\title{
L'évaluateur et la sagesse pratique : vecteurs essentiels pour assurer la crédibilité de l'évaluation
}

\author{
Sylvain Houle, Marthe Hurteau et Marie-Pier Marchand \\ Université du Québec à Montréal
}

\begin{abstract}
Résumé : Le présent article revisite les données recueillies dans le cadre d'une recherche antérieure conduite par Hurteau et Houle (2008). À l'époque, les chercheurs n'avaient pas été en mesure de détailler la contribution des compétences personnelles de l'évaluateur au pilotage d'une évaluation dont les résultats sont acceptables pour les parties prenantes et d'en effectuer une analyse exhaustive, faute d'un cadre théorique adéquat. En introduisant le concept de sagesse pratique, House (2015) leur offre l'opportunité de le faire. Cet auteur définit le concept comme «... doing the right thing in the special circumstances of performing the job » (p. 88). De plus, Schwartz et Sharpe (2010) offrent des critères qui permettent d'en établir la présence. La démarche actuelle a consisté à analyser le témoignage d'un policier d'expérience. Les résultats ont permis de bonifier notre compréhension du concept, tout en soulignant la contribution du Télos (valeurs morales) ainsi que de critères supplémentaires, tels que l'importance d'un temps de réflexion et la nécessité de compléter les informations lorsque cela s'avère nécessaire.
\end{abstract}

Mots clés : jugement, pratique évaluative, sagesse pratique

\begin{abstract}
This article revisits data collected during previous research conducted by Hurteau and Houle (2008). At the time, the researchers were not able to account for the evaluator's personal skills to pilot an evaluation, the results of which were acceptable to the stakeholders, and to conduct a comprehensive analysis, for lack of an appropriate theoretical framework. By introducing the concept of practical wisdom, House (2015) offers them the opportunity to do so. The author defines this concept as "... doing the right thing in the special circumstances of performing the job" (p. 88). In addition, Schwartz and Sharpe (2010) offer criteria used to establish its presence. The current approach consisted of analyzing the testimony of an experienced police officer. The results helped to improve our understanding of the concept, highlighting the contribution of Telos (moral values) as well as additional criteria such as the importance of time for reflection and the need to supplement the information where necessary.
\end{abstract}

Keywords: judgment, evaluative practice, practical wisdom 


\section{INTRODUCTION}

La crédibilité demeure une dimension fondamentale qui préoccupe plusieurs praticiens dans le domaine de l'évaluation de programme. À cet effet, la recherche de Hurteau et Houle (2008) avait pour objet d'étude la description du processus sous-jacent à l'élaboration d'un jugement crédible, c'est-à-dire recevable par lensemble des parties prenantes. Les résultats ont mis en évidence la présence de différents critères permettant de favoriser cette " crédibilisation » par les parties prenantes, dont la contribution des dispositions personnelles de lévaluateur. Or, comme le mentionne Perrin (2010), les compétences plus soft ont souvent tendance à être ignorées, situation qui peut être attribuable au fait que les évaluateurs ne disposent pas d'un cadre de référence théorique à cet égard.

Cet article vise à partager la réflexion que nous avons réalisée à la suite de la lecture du récent ouvrage de House (2015), dans lequel l'auteur traite d'un concept lié aux dispositions personnelles de l'évaluation, soit celui de la sagesse pratique. Nous y avons perçu un rapprochement évident avec certains témoignages recueillis dans le cadre de la recherche initiale de Hurteau et Houle (2008). Ces témoignages ont soudainement pris une tout autre dimension. Cest ainsi que nous avons décidé de revisiter le verbatim des entretiens effectués lors de cette étude et de reprendre l'analyse des données à la lumière des derniers développements proposés par House (2015) quant au concept de sagesse pratique. Cette nouvelle analyse adopte une perspective exploratoire et descriptive, visant à décrire et bonifier notre compréhension du concept. De plus, si House (2015) note que « ... being a good evaluator doesn't depend solely on plans and methods» (p. 87), tout en ajoutant que "... evaluators evaluate with their whole person, not only with methods" (p. 127), ne devient-il pas pertinent de mieux saisir la contribution de la sagesse pratique afin de bonifier notre compréhension de la pratique?

Dans les sections qui suivent, nous exposons le fruit de notre démarche. Nous y proposons d'abord d'explorer le concept de sagesse pratique, puis nous y décrivons plus amplement notre démarche sur le plan méthodologique. Les résultats ayant émergé sont ensuite présentés et discutés.

\section{CONCEPT DE SAGESSE PRATIQUE}

\section{Origine et définition}

Aristote a été un des premiers à remarquer que les ouvriers n'appliquaient pas systématiquement des règles rigides et que le succès de leurs travaux résidait justement dans leur capacité à sảapter aux différents contextes et différentes situations (Schwartz et Sharpe, 2010). Cette observation est à l'origine du concept de sagesse pratique que Saugstad (2002) définit comme « ... an ability to discern the salient particulars of a situation (to size up the situation) and to understand what general knowledge, principles, and values are involved in deciding what to do on a particular occasion » (p. 98). Il est important de préciser que la sagesse pratique s'inscrit dans la perspective de porter un jugement, de prendre une décision et d’agir et non pas 
d'uniquement réfléchir, discourir. Pour House (2015), elle consiste à « ... doing the right thing in the special circumstances of performing the job. Professional practices, including evaluation, also require such choices " (p.88). Lauteur illustre son propos par le témoignage de Luke (tiré de Schwartz et Sharpe, 2010) : Luke fait partie de l'équipe d'entretien de chambres dans un centre hospitalier. Il rapporte le cas d'un patient plongé dans le coma depuis plusieurs semaines à la suite d'une bagarre. Son père, qui est fréquemment à son chevet, interpelle Luke insatisfait du ménage de la chambre. Luke lui répond qu'il a pourtant procédé comme d'habitude, mais le père demeure sur ses positions. Plusieurs options soffrent à Luke : ignorer le père, lui répondre poliment et vaquer à ses occupations, argumenter avec celuici, faire appel à son superviseur... Mais, contre toute attente, il choisit de refaire le ménage, mettant ainsi fin à la polémique. Par la suite, Luke justifie son choix : il a compris que la réaction du père nétait pas tant attribuable au ménage, comme au fait qu'il était soumis à un stress depuis plusieurs semaines. En agissant ainsi, il espérait que le père aurait l'impression de contrôler " quelque chose ", puisque cela nétait pas le cas pour la santé de son fils. C'est ce qui fait dire à House (2015) qu'une telle réaction est empreinte de sagesse pratique, Luke ayant fait "... what makes the situation better, instead of merely doing what's in the job description.» (p. 88).

Ainsi, un professionnel gagne à effectuer une lecture complète de l'événement afin d'agir adéquatement. Et le lecteur comprendra que le recours à la sagesse pratique va au-delà de l'application d'une procédure, pour faire appel à des aptitudes particulières qui favorisent l'adoption de la stratégie requise, dans un contexte spécifique, et qui est axée sur le succès de la démarche (Saugstad, 2002).

\section{Caractéristiques}

Quelles sont les caractéristiques que doit revêtir un professionnel pour faire preuve de sagesse pratique? Schwartz et Sharpe (2010) les systématisent sous la forme de six critères qui doivent être satisfaits par celui-ci. Plus précisément, il doit : 1) comprendre le but poursuivi et poser les gestes qui contribuent à sa réussite; 2 ) improviser, balancer des objectifs contradictoires et interpréter les règles à la lumière des particularités de chaque contexte; 3) faire preuve de perspicacité en effectuant une lecture intelligente des contextes sociaux et en saisissant les nuances et des subtilités qui les distinguent; 4) élucider les différentes perspectives adoptées par les personnes concernées, envisager la situation selon leurs points de vue et capter ce que ressent une autre personne (empathie); 5) utiliser ses émotions pour comprendre ce qui se passe et poser un jugement éclairé (intuition bien éduquée); et 6) avoir acquis une expérience dans son domaine, ce qui amène l'auteur à conclure qu'on apprend à être brave en posant des gestes courageux, sous-entendant quon apprend aussi, et surtout, de ses erreurs.

En résumé : "practical wisdom is not about establishing rules and following them. It's about if, when, and how to apply the rules" (House, 2015, p. 90). Cet auteur identifie aussi deux conditions requises pour que le recours à la sagesse pratique s'avère efficace, soit : 1 ) un environnement stable et régi par des protocoles 
ou processus clairs et 2) loccasion de tirer des apprentissages de la pratique grâce à une régularité des événements qui favorise la mise en place d'un processus de reconnaissance. House considère que la plupart des évaluations sont en mesure de répondre à ces conditions. Cependant, il met aussi les praticiens en garde contre un éventuel « excès de confiance » qui s'installe lorsque ceux-ci ont tellement l'impression de "reconnaître » un événement qu'ils omettent d'identifier les spécificités de chaque situation.

\section{Contribution de Télos}

La sagesse pratique commande d'être soutenue par Télos, c'est-à-dire par des

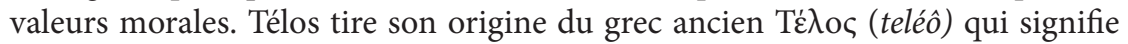
fixer une cause finale. Ce terme neutre se définit comme une morale pratique s'inscrivant dans la volonté de trouver une bonne solution. Télos se manifeste lorsque la personne est en mesure d'extraire l'essentiel d'une situation à travers un ensemble de bruits, d'envisager les options qui soffrent à elle et de choisir celle qui s'avère la plus optimale dans les circonstances. Dans ce sens, l'expérience et l'expertise de la personne s'avèrent de précieux alliés parce qu'elles lui permettront d’envisager les conséquences de ses actes.

Télos constitue ainsi les fondements de la sagesse pratique en guidant la démarche afin qu'elle présente les bons choix et la bonne solution.

Et cela s'avère particulièrement précieux lorsqu'il faut balancer des buts en concurrence. Schwartz et Sharpe (2010) illustrent le présent propos de la façon suivante : l'enseignant enseigne et inspire; l'avocat fournit de bons services juridiques et sert la justice; le médecin soigne et fait preuve de compassion et d'honnêteté. Ainsi, en plus d'assurer l'entretien des chambres, Luke est totalement dédié à fournir un soutien émotionnel aux patients et à leurs familles afin qu'ils se sentent aussi bien que possible dans les circonstances et qu'ils gardent espoir.

\section{MÉTHODOLOGIE}

La démarche que nous avons réalisée s'inscrit dans une perspective exploratoire visant à effectuer une nouvelle analyse des données recueillies lors de la recherche de Hurteau et Houle (2008) en revisitant les verbatim existants. Dans un premier temps, nous effectuons une brève présentation de la démarche initiale afin de permettre au lecteur de saisir son contexte et d'établir la pertinence de ces données. Nous enchainons en second lieu par une description de la démarche actuelle.

\section{Description de la démarche initiale (Hurteau et Houle, 2008)}

La recherche initiale s'intéressait au processus conduisant à lélaboration d'un jugement crédible en évaluation de programme. Cette recherche qualitative s'inscrivait dans une perspective de la grounded theory. Les chercheurs ont réalisé des entretiens individuels non structurés qui consistaient à demander aux participants de relater deux situations issues de leur pratique professionnelle : une dans laquelle ils jugeaient avoir posé un «bon » jugement et une autre situation où ils considéraient 
avoir posé un «moins bon » jugement. Par la suite, ils étaient invités à comparer les deux situations afin d'identifier ce qui les distinguait.

S’appuyant sur la prétention de Stake (2011) voulant qu'un jugement professionnel ne soit pas l'apanage d'une discipline, un échantillonnage intentionnel a été retenu. Celui-ci était composé de dix-neuf professionnels provenant de divers horizons, ayant au moins cinq ans d'expérience et étant appelé à porter un jugement sur une base régulière dans l'exercice de leur fonction. Les entretiens ont été enregistrés et retranscrits par la suite sous la forme d'un verbatim. Les chercheurs ont eu recours au logiciel Nvivo pour effectuer une analyse de façon individuelle. Par la suite, ils ont mis en commun et confronté leurs résultats, sous la supervision d'un expert externe spécialisé dans l'approche analytique de la grounded theory, afin de dégager un consensus entre les chercheurs. Le consensus obtenu met en lumière différents principes et critères permettant de décrire le processus inhérent à l'élaboration d'un jugement crédible.

\section{Description de la démarche actuelle}

Rappelons que la présente démarche vise à décrire des manifestations de sagesse pratique afin de mieux comprendre sa contribution dans le cadre de la production d'un jugement qui se veut crédible. Pour ce faire, nous avons effectué une analyse de cas, lequel a été ciblé sur une base intentionnelle de par la richesse et la pertinence qu'il présentait pour approfondir le concept de sagesse pratique (purposeful sampling, single significant case) (Patton, 2015). Plus précisément, notre attention s'est portée sur le verbatim d'un policier d'expérience. En plus de relater deux actions qui conduisent respectivement à la production d'un jugement positif et d'un jugement négatif, le policier fournit spontanément une réflexion qui facilite l'approfondissement et l'enrichissement de la compréhension du concept ainsi que l'élaboration d'idées percutantes.

Lanalyse des données s'appuie sur les critères énoncés par Schwartz et Sharpe (2010), entérinés par House (2015), sur la description du concept Télos et sur l'émergence de critères qui contribuent à bonifier notre compréhension du concept de sagesse pratique.

\section{RÉSULTATS}

Les résultats permettent de classer des segments des verbatim en fonction de critères prédéterminés, comme la contribution de Télos et ceux énoncés par Schwartz et Sharpe (2010) qui confirment la manifestation de sagesse pratique. À cela, s'ajoute un critère émergent, soit la contribution de dimensions liées au temps de réflexion et à la validation de l'information.

\section{Contribution de Télos}

Télos constitue le fondement de la réflexion et de l'inspiration. Il trouve ses manifestations dans les valeurs qui guident l'action. D’entrée de jeu dans le cadre de son entretien, le policier annonce un principe qui guide ses actions : 
Je vais vous donner un principe que je me suis donné, les gens en général font très rarement affaire avec la police, donc jessaie de donner une bonne image de mon métier. Je me fais un devoir d'essayer dêtre professionnel, je vais essayer dêtre poli, courtois, c'est certain que si jai à être directif parce que la situation le commande, je vais lêtre, mais je me suis toujours donné comme directive de faire cela... Je connais des policiers qui ne veulent pas se casser la tête, qui disent moi non... qui vont prendre des décisions en se basant uniquement sur leur code de déontologie et pour les assises légales, c'est super facile...

\section{Il poursuit :}

Je pense qu’on a le droit à l’erreur, à un moment donné. On a le droit de ne pas tout savoir. Il y a des événements que j’ai vécus à répétition où je suis très à l'aise, mais il $\mathrm{y}$ a aussi d'autres événements qu'on voit moins souvent. Mais il y a quand même une routine qui s'installe dans le milieu policier. C'est quand même souvent le même genre d'appel. Mais des fois, on a un appel qui nous sort de notre routine, de notre confort, et à ce moment, on n'a pas tous les éléments pour pouvoir prendre une bonne décision... Il faut être capable de temps en temps de se dire, oups, minute, tu es dans ta zone de confort, il faut que tu sortes de là un petit peu... Ce n'est jamais pareil comme la dernière fois. Oui il y a une certaine routine, mais il y a toujours des nuances et c'est tout le temps cette nuance-là qui est dangereuse. On ne peut jamais prendre une décision en pensant à l'autre fois. Il y a toujours un petit quelque chose de nouveau, une donnée qu'on n'avait pas qui s'est rajoutée... il y a toujours un petit quelque chose qui peut venir changer la décision complètement. Autant positif que négatif.

Nous verrons dans les expériences rapportées comment il s'inspire, ou non, de ces principes personnels dans sa gestion des événements.

\section{Contribution des critères énoncés par Schwartz et Sharpe (2010)}

Dans un premier temps, nous présentons des segments de verbatim concernant le témoignage recueilli auprès du policier. Il nous relate deux événements qui ont conduit à des jugements et des décisions, le premier est envisagé comme positif alors que le second s'avère négatif. Leur mise en parallèle permet d'illustrer les critères énoncés par Schwartz et Sharpe (2010) et de contribuer à notre compréhension de concept de sagesse pratique. Ceux-ci ont été développés antérieurement.

Extrait du verbatim relatant le cas positif :

La situation c'est il y a quelques mois, j'intercepte un véhicule parce que c'est un véhicule désuet, un vieux véhicule, la personne qui est à l'intérieur c'est un homme d'une quarantaine d'années, avec trois enfants à l'intérieur. Je fais une enquête rapide de la plaque d'immatriculation, et du permis de conduire. Le monsieur est sanctionné, il n'a pas de permis. Donc il n'a pas le droit de conduire. Il a perdu son permis en juin, sous toute réserve, pour des amendes non payées. Donc la loi ce qu'elle nous dit dans ce cas-là ce nest pas compliqué, c'est la saisie du véhicule pour une durée de 30 jours, c'est un constat d'infraction de 438 \$, et c'est le remorquage du véhicule. Donc on parle de frais de $1000 \$$. Là on se dit, le gars a peut-être du mal à arriver, qu'est-ce qu'on fait dans 
ce cas-là. Il y a certains policiers qui ne se cassent pas la tête, ils suivent la loi. Donc je discute avec le monsieur qui est bien correct, il m'explique qu'il n'a pas l'habitude de prendre le véhicule. Le véhicule est plaqué. Les plaques sont bonnes et il est au nom de sa conjointe. Il dit que ce matin il avait une urgence. Il allait à la clinique avec le petit, sa femme est au travail, que normalement il ne le prend jamais le véhicule. Et effectivement, il y a un petit bébé dans un banc et on voit qu'il est malade. Et je l'intercepte pas loin d'une clinique. Donc dans cette situation-là, je n’ai pas remisé le véhicule. Je n'ai pas donné de constat au monsieur. Je ne l'ai pas fait parce que je me disais que ce serait les enfants qui allaient en payer le prix et sa conjointe aussi. Si j’enlève le véhicule à cette famille-là pendant un mois, ils vont souffrir. Je me dis que ça ne sert à rien de lui taper sur la tête encore plus. J'ai quand même validé des choses, j'ai quand même validé avec sa conjointe, elle m’a confirmé tout cela, j’ai validé aussi avec lui qui allait vraiment chez le médecin, parce qu'il aurait pu me conter n'importe quoi. C'est un petit peu une chance qu'on prend, je lui laisse le bénéfice du doute, il est correct avec moi, un peu dans la misère, c'est un peu mes valeurs humaines qui rentrent en ligne de compte. J'ai un pouvoir discrétionnaire. La loi me permet de prendre une décision, d'y aller selon mon bon sens là-dedans, et j’ai laissé aller le monsieur. Je lui ai dit d'aller à la clinique, de demander à sa conjointe d'aller chercher le véhicule, je lui ai dit que je ne voulais plus le voir conduire. Le monsieur est content, il n'a pas d'autre choix dêtre content parce que je lui ai sauvé beaucoup de troubles. Et où jai été content d’avoir pris cette décision-là, c'est de l'avoir croisé ce monsieur-là, quelques semaines après, et il était assis côté passager, c'est sa femme qui conduit. Et je l'ai intercepté par curiosité, voir si effectivement ce nétait pas lui qui conduisait, puis non, il m’a dit, je ne conduis pas, je vous l'ai dit, c'était une urgence qui a fait que je suis allé à la clinique, j’ai apprécié votre geste, j’ai apprécié la chance que vous m’avez donnée, et ça ne me tente pas de me refaire prendre et de tomber sur quelqu'un qui ne me donnerait pas nécessairement cette chance-là, et je m’enfoncerais encore plus...

\section{Voici maintenant un extrait du verbatim relatant le cas négatif :}

L'autre situation, c'est justement ma zone de confort qui m’a influencé beaucoup dans cette décision-là... C'était un dimanche matin... Un appel rentre, une chicane de famille, dans un village de Lanaudière. C'est un endroit où je suis allée maintes et maintes fois... On est au resto et c'est mon secteur donc je dis aux gars, dérangez-vous pas, je vais y aller seul, c'est rien de grave. Je sais c'est qui, je suis allé quelques fois. En partant, mon jugement était influencé parce que je les connaissais, j’étais dans ma zone de confort. Parce que jétais déjà allé là. En partant, j’avais jugé cela comme une situation facile... Cétait un endroit très loin du poste, 30 minutes à vitesse urgente. Arrivé sur place, lui est dans la cuisine, elle aussi, il lui tient le bras et il a un couteau à la main. Il la retient. En partant, ce nétait pas comme avant. Ma décision était mauvaise, et quand lui voit la police arrivée, son agressivité monte en flèche. Mon intervention banale n'est plus banale, c'est une intervention à haut risque et je suis seul. Et mon backup est à 30 minutes. Donc je dois intervenir. La prise de décision au départ, j’ai mal jugé la situation. Par la suite j’ai réussi à rattraper en discutant avec lui, en apportant une diversion, en attirant son attention vers moi, il a réussi à la lâcher, elle est sortie par derrière donc elle était sécurisée. Ensuite, il y a eu du poivre de Cayenne dans la maison, et j’ai réussi à sécuriser tout le monde, à faire un périmètre de sécurité, à mettre cela sous contrôle, mais quand mes confrères sont arrivés, on a dû négocier 
pendant 3-4 heures. On a réussi à rattraper l'histoire... On aurait dû y aller à deux... Quand je suis assis une heure ou deux après l'événement et que j'écris le rapport, je me tape dans le front et je me dis, maudit épais, innocent. Qu'est-ce que tu as fait là. Tu le sais que ça peut mal virer.

Le Tableau 1 permet de faire ressortir les différences entre les deux situations en fonction des critères retenus.

Ainsi, si le cas positif peut aisément se conformer à l'ensemble des critères, il est évident qu'il n'en est pas de même pour le cas négatif. Si l'on met de côté le caractère dramatique que revêt le cas négatif, il n'en demeure pas moins qu'il reflète une situation fréquente, soit celle de faire une association avec des expériences antérieures - contrairement à la démarche d'identifier le contexte particulier - et de rentrer ainsi dans sa zone de confort parce qu'on avait alors trouvé une solution appropriée. Il est à noter que le policier fait preuve de perspicacité dans le cas négatif, mais seulement après coup. Cela confirme les propos des théoriciens qui affirment qu'on apprend autant de nos erreurs que de nos succès.

Or, en plus de se conformer aux critères élaborés par Schwartz et Sharpe (2010), la sagesse pratique exige un temps de réflexion et une validation d'informations, comme nous le verrons au point suivant.

\section{Critères supplémentaires : temps de réflexion et validation d'informations}

La sagesse pratique requiert un temps de réflexion et la validation d'informations qui savèrent parfois complémentaires. Les propos du policier appuient ce constat :

On doit toujours prendre un minimum de données et faire un minimum de vérification pour pouvoir prendre cette décision-là... Le temps nous permet d'avoir le plus de données possible... Où qu’on prenne de mauvais jugements, c'est souvent quand on manque de données... il faut aller chercher d'autres informations, ou il faut valider avec quelqu'un... Dans la police il y a beaucoup de zones grises. Il y a tout le temps des nuances. Et on a la chance d'aller valider plus haut quand on a le temps... Quand on doit prendre une décision rapide, les valeurs sont souvent mises de côté.

Il argumente son point en reprenant l'exemple négatif :

Avant même d'avoir vu la situation, je me suis fié à des expériences que j’avais eues là-bas, je connaissais le genre de personnes, et c'est à ne pas faire. C'est après qu'on se rend compte de tout cela. Je peux vous dire que maintenant une chicane de famille, je prends cela au sérieux. Même si dans $99,9 \%$ des cas, on réussit à régler cela correctement... Cette expérience-là négative m’aide maintenant, mais toutes les expériences que javais eues avant cet événement-là mont nui un peu. Ça m’a permis d'acquérir du confort, d'acquérir du confort dans certains événements.

En résumé, la manifestation de la sagesse pratique requiert la présence de Télos qui assure un encadrement à l'action par des valeurs morales; elle doit aussi se conformer aux critères énoncés par Schwartz et Sharpe (2010), à savoir que la 
Tableau 1. Conformité aux critères énoncés par Schwartz et Sharpe (2010)

\begin{tabular}{|c|c|c|}
\hline Critères & Cas positif & Cas négatif \\
\hline $\begin{array}{l}\text { Comprendre le but et } \\
\text { y contribuer }\end{array}$ & $\begin{array}{l}\text { Le monsieur est sanctionné, il } \\
\text { n'a pas de permis. Si j'enlève } \\
\text { le véhicule à cette famille-là } \\
\text { pendant un mois, ils vont souf- } \\
\text { frir. Je me dis que ça ne sert } \\
\text { à rien de lui taper sur la tête } \\
\text { encore plus. }\end{array}$ & $\begin{array}{l}\text { Un appel rentre, une } \\
\text { chicane de famille... C'est } \\
\text { un endroit où je suis allée } \\
\text { mainte et mainte fois... }\end{array}$ \\
\hline $\begin{array}{l}\text { Improviser, balancer } \\
\text { des objectifs contra- } \\
\text { dictoires et interpréter } \\
\text { les règles à la lumière } \\
\text { des différents con- } \\
\text { textes }\end{array}$ & $\begin{array}{l}\text { Il allait à la clinique avec le petit, } \\
\text { sa femme est au travail, que } \\
\text { normalement il ne le prend } \\
\text { jamais le véhicule... Donc dans } \\
\text { cette situation-là, je n'ai pas } \\
\text { remisé le véhicule. Je n'ai pas } \\
\text { donné de constat au monsieur. }\end{array}$ & $\begin{array}{l}\text { Je vais y aller seul, ce n'est } \\
\text { rien de grave. Je sais c'est } \\
\text { qui, je suis allé quelques } \\
\text { fois. }\end{array}$ \\
\hline Être perspicace & $\begin{array}{l}\text { J'ai un pouvoir discrétionnaire. } \\
\text { La loi me permet de prendre } \\
\text { une décision, d'y aller selon } \\
\text { mon bon sens là-dedans, et j'ai } \\
\text { laissé aller le monsieur. }\end{array}$ & $\begin{array}{l}\text { Quand je suis assis une } \\
\text { heure ou deux après } \\
\text { l'événement et que j'écris } \\
\text { le rapport, je me tape } \\
\text { dans le front et je me dis, } \\
\text { maudit épais, innocent. } \\
\text { Qu'est-ce que tu as fait } \\
\text { là. Tu le sais que ça peut } \\
\text { mal virer. }\end{array}$ \\
\hline $\begin{array}{l}\text { Élucider les différentes } \\
\text { perspectives }\end{array}$ & $\begin{array}{l}\text { J'ai quand même validé des } \\
\text { choses... parce qu'il aurait pu } \\
\text { me conter n'importe quoi. }\end{array}$ & $\begin{array}{l}\text { En partant, mon juge- } \\
\text { ment était influencé parce } \\
\text { que je les connaissais, } \\
\text { j'étais dans ma zone de } \\
\text { confort. Parce que j'étais } \\
\text { déjà allé là. En partant, } \\
\text { j'avais jugé cela comme } \\
\text { une situation facile... }\end{array}$ \\
\hline Être empathique & $\begin{array}{l}\text { C'est un petit peu une chance } \\
\text { qu'on prend, je lui laisse le } \\
\text { bénéfice du doute, il est cor- } \\
\text { rect avec moi, un peu dans } \\
\text { la misère, c'est un peu mes } \\
\text { valeurs humaines qui rentrent } \\
\text { en ligne de compte. }\end{array}$ & \\
\hline
\end{tabular}

personne : 1) comprend le but poursuivit et elle désire poser les gestes qui contribuent à sa réussite; 2 ) peut improviser, balancer des objectifs contradictoires et interpréter les règles à la lumière des particularités de chaque contexte; 3 ) savère perspicace par une lecture intelligente des contextes sociaux et une saisie des 
nuances et des subtilités qui les distinguent; 4) élucide les différentes perspectives adoptées par les personnes concernées, envisage la situation selon leurs points de vue et capte ce que ressent une autre personne (empathie); et 5) a recours à ses émotions pour comprendre ce qui se passe et poser un jugement éclairé. De plus, la manifestation de sagesse pratique requiert un temps de réflexion ainsi que la validation d'informations.

\section{DISCUSSION}

Patton (1986) associe la réussite d'une évaluation à un mélange d'acteurs, de jeux politiques, d'histoire, de contextes, de ressources, de contraintes, de valeurs, de besoins, de préférences et de chance. House (2015) confirme ces propos, tout en nous rappelant qu'il faut toujours avoir à l'esprit que : « ... evaluators seriously underestimate how long it takes for people to assimilate bad news» (p. 116). Pour cet auteur, la sagesse pratique peut contribuer de façon significative en rendant le jugement plus juste et acceptable, c'est-à-dire crédible, aux yeux des parties prenantes. Ce simple constat peut faire la différence entre une démarche qui s’avère un succès, d'une autre qui s’avère un échec.

Pour y parvenir, le professionnel doit entreprendre une analyse constante du contexte afin d'effectuer les ajustements requis par la situation en présence et sans pour autant dénaturer le protocole prévu. Il doit aussi faire preuve d'empathie envers les acteurs. Le jugement qui émane de la démarche pourrait aussi faire l’objet d'une validation auprès de pairs, mais en tout temps il doit être formulé dans le respect des personnes concernées. Pour sa part, l' « échec », ou la formulation d'un jugement non crédible, semble se caractériser par un manque d'informations ou une mauvaise gestion de l'information, par une application trop rigide des protocoles, par un manque d'empathie ou par un "excès de confiance ", attribuable à une « fausse » reconnaissance de situations antérieures familières. Par ailleurs, il ne faut pas négliger la contribution de Télos, tel que fut le cas dans l'exemple d'un jugement négatif rapporté par le policier.

Il est important de souligner que la sagesse pratique ne constitue pas une panacée à tous les problèmes et les défis rencontrés dans le cadre d'une démarche évaluative. Cependant, elle peut contribuer significativement à formuler la meilleure solution dans un contexte précis. En effet, le succès ou léchec dans la production d'un jugement se résume à peu de choses : une mauvaise appréciation d'un fait dans le déroulement des opérations, une information qui apparait incomplète. House (2015) illustre ce propos en relatant le cas d'un litige concernant la présentation des résultats d'une évaluation dans le rapport et sa gestion de lévénement. Le client nétait pas content parce qu'il considérait que celui-ci pouvait prêter à confusion: "... Our analysis shows the gains. Then we add another page that shows no gains, on the next page. Readers would have both. Would that satisfy you?". Et House (2015) d'ajouter : "I think we can live with that... " (p. 119). Ainsi, en acceptant la suggestion du client, ils sont sortis de l'impasse qui compromettait toute la démarche évaluative, et ce, il importe de le souligner, sans pour autant 
compromettre l'intégrité du rapport. Geste simple, mais combien déterminant et empreint de sagesse.

Dans ce sens, lévaluateur constitue un vecteur essentiel de la démarche parce qu'il est responsable de la co-construction du jugement tout au long du parcours en collaboration avec les parties prenantes. Pour y parvenir, il doit être en mesure d'évaluer le déroulement, d'effectuer les ajustements qui s'imposent, mais sans pour autant "faire n'importe quoi pour plaire " (Smith, 2011) ou dénaturer le processus. Ces constats nous ramènent aux définitions de la sagesse pratique émise par Saugstad (2002) et House (2015). En effet, les deux auteurs l'envisagent comme la capacité de saisir les particularités d'une situation afin de « faire ce qu'il se doit pour réussir ».

Finalement, il ne faut surtout pas oublier que cette quête d'une solution qui respecte l'intégrité de la démarche, tout en respectant les acteurs, s'appuie sur un fondement peu apparent, mais combien essentiel, soit la présence de valeurs (Télos). Les propos rapportés par le policier vont dans le même sens que ceux tenus par Schwartz et Sharpe (2010) en nous révélant qu'il lui apparait important de donner une bonne image de la police face à la population et dêtre nuancé dans ses décisions, surtout lorsque le code lui accorde un pouvoir discrétionnaire. Non seulement met-il ses principes en application, mais il va au-delà : « donc dans cette situation-là, je n’ai pas remisé le véhicule. Je n’ai pas donné de constat au monsieur. Je ne l'ai pas fait parce que je me disais que ce serait les enfants qui allaient en payer le prix et sa conjointe aussi. Si jenlève le véhicule à cette famille-là pendant un mois, ils vont souffrir. Je me dis que ça ne sert à rien de lui taper sur la tête encore plus. » Et ce, soulignons-le, toujours dans le respect des lois puisqu'il a un droit discrétionnaire dans cette situation.

\section{FORCES ET LIMITES}

La présente démarche s'appuie sur des données secondaires, soit le verbatim recueilli dans le cadre de la recherche initiale de Hurteau et Houle (2008). Nous étions conscients des contraintes liées à un tel choix méthodologique. Cependant, nous avons été en mesure de démontrer la rigueur dans laquelle l'information a été recueillie et traitée ainsi que sa pertinence. Enfin, la présentation d'un seul cas pourrait être considérée comme restrictive, mais il ne faut pas oublier que le but du présent exercice nest pas tant de démontrer la fréquence d’apparition de manifestations de sagesse pratique comme d'en bonifier sa compréhension.

\section{CONCLUSION}

La présente démarche permet de mieux comprendre ce concept qui peut sembler abstrait, soit celui de sagesse pratique et, par le fait même, de contribuer au développement d'une théorisation de l'acte dévaluer. En effet, les données mettent en évidence la contribution de Télos (valeurs morales) et elles confirment la pertinence des critères proposés par Schwartz et Sharpe (2010). Les données 
soulignent l'importance d'un temps de réflexion et la nécessité de compléter les informations lorsque cela s'avère nécessaire. Finalement, nous ne pouvons faire autrement que de conclure en mettant en exergue le rapprochement que nous effectuons entre la sagesse pratique et l'éthique professionnelle. En effet, la Commission de l'éthique en science et en technologie (2013) ne définit-elle pas l'éthique comme « ... un ensemble de valeurs ni de principes en particulier. Il s'agit d'une réflexion argumentée en vue du bien-agir. Elle propose de s'interroger sur les valeurs morales et les principes moraux qui devraient orienter nos actions, dans différentes situations, dans le but d'agir conformément à ceux-ci ». Dans ce sens, tant la sagesse pratique que l'éthique font appel à une pensée réflexive pour éclairer la prise de décision.

\section{RÉFÉRENCES}

Commission de l'éthique en science et en technologie. (2013). Éthique. Consulté le 11 décembre 2017 au http://www.ethique.gouv.qc.ca/fr/ethique.html

House, E.R. (2015). Evaluating: Values, biases, and practical wisdom. Charlotte, NC: Information Age Publishing Inc.

Hurteau, M. et Houle, S. (2008). Mieux comprendre la méthodologie requise à la production d'un jugement crédible en évaluation de programme. Recherche subventionnée par le Conseil de la recherche en sciences humaines du Canada (CRSH).

Patton, M.Q. (1986). Utilization-focused evaluation. 2e édition. Thousand Oaks, CA: Sage.

Patton, M.Q. (2015). Qualitative research and evaluation methods. 4e édition. Los Angeles: Sage Publications.

Perrin, B. (2010). Learning from evaluation misadventures: The importance of good communication [numéro spécial]. Revue canadienne en évaluation de programme, 25, $115-124$

Saugstad, T. (2002). Educational theory and practice in an Aristotelian perspective. Scandinavian Journal of Educational Research, 46(4), 373-390. https://doi. org/10.1080/0031383022000024561

Schwartz, B. et Sharpe, K. (2010). Practical wisdom: The right way to do the right thing. New York: Penguin Group.

Smith, N.L. (novembre 2011). Emergent, investigative evaluation: Theory, development, and use in evaluation practice. Colloque annuel de l'American Evaluation Association, Anaheim.

Stake, R.E. (mai 2011). Evaluation's generations: Veneration, vituperation, or simply divestiture. Conférence d'ouverture du colloque annuel de la Société canadienne dévaluation, Edmonton, Canada.

\section{PRÉSENTATION DES AUTEURS}

Sylvain Houle, Ph. D., est professeur au département des sciences comptables à l'École des sciences de gestion à l'Université du Québec à Montréal (UQAM). Son doctorat portait sur lévaluation de programme et il a toujours gardé un intérêt pour le domaine. 
Marthe Hurteau, PH. D., est professeure titulaire au département d'éducation et pédagogie, Faculté des sciences de l'éducation de l'Université du Québec à Montréal (UQAM). Elle détient le titre dévaluatrice accréditée décerné par la Société canadienne d'évaluation (SCE).

Marie-Pier Marchand est doctorante en éducation à l'Université du Québec à Montréal (UQAM). Dans le cadre de sa thèse, elle s'intéresse à la contribution des différents groupes de parties prenantes au sein des démarches d'évaluation. 